\title{
SIMULAÇÃO DE PACIENTES - CURSOS DE SUPORTE DE VIDA Suporte Avançado de Vida no Trauma
}

\author{
SIMULATION OF PATIENTS - LIFE SUPPORT COURSES \\ ADVANCED TRAUMA LIFE SUPPORT
}

Sandro Scarpelini ${ }^{1}$, José Ivan de Andrade ${ }^{2}$

\begin{abstract}
${ }^{1}$ Docente. Disciplina de Cirurgia de Urgência e Trauma. Departamento de Cirurgia e Anatomia. Faculdade de Medicina de Ribeirão Preto - USP. ${ }^{2}$ Docente Aposentado. Departamento de Cirurgia e Anatomia. Faculdade de Medicina - USP. Docente. Curso de Medicina. Centro Universitário Barão de Mauá - Ribeirão Preto.

CorResPondência: Sandro Scarpelini (sandro@fmrp.usp.br). Centro de Estudos de Emergências em Saúde. Rua Bernardino de Campos, 1000, 2 andar. 14015-130 - Ribeirão Preto - SP.
\end{abstract}

Scarpelini S, Andrade JI. Simulação de pacientes - Cursos de Suporte de Vida - Suporte Avançado de Vida no Trauma. Medicina (Ribeirão Preto) 2007; 40 (2):199-203.

Resumo: Durante os últimos 30 anos, o curso "Advanced Trauma Life Support" - Suporte Avançado de Vida no Trauma, do American College of Surgeons, tornou-se a principal referência mundial no ensino do atendimento inicial ao traumatizado. A partir de uma primeira experiência em Nebraska, em 1978, o curso difundiu-se rapidamente para mais de 25 países, graças a sua abordagem objetiva, prática e dinâmica do ensino das prioridades vitais do traumatizado grave e por sistematizar algumas técnicas cirúrgicas indispensáveis para o sucesso do atendimento. Este modelo de ensino, baseado em simuladores artificiais, em modelos animais e atores humanos se apresenta como alternativa eficiente para o ensino de emergências médicas.

Descritores: Educação Médica. Simulação. Trauma. SAVT. Suporte Avançado de Vida no Trauma.

"O avião caiu entre as árvores... Minha esposa (32 anos) foi arremessada a $90 \mathrm{~m}$ de distância, e morreu instantaneamente... Christopher (10 anos), Richard (8 anos), Randal (7 anos) e Kimberly (3 anos), nossos filhos, sofreram lesões gravíssimas... Horas após o acidente, nós chegamos ao hospital como uma tropa arrasada, roupas rasgadas, enlameadas e úmidas, com as faces ensangüentadas. Fomos para a porta da sala de emergência. Ela estava trancada. Batemos na porta, a enfermeira de plantão respondeu... Ela informou que teríamos que esperar até os médicos chegarem..." 1.

Após esta experiência, em 1976, o Dr. James Styner, um ortopedista de Nebraska, mobilizou-se e com a ajuda de outros médicos e enfermeiros da Lincoln Medical Education Foundation e da Universidade de Nebraska criou o curso "Advanced Trauma Life Support" (ATLS). Nesta época, nos EUA, o principal argumento do Dr. Styner foi "Quando eu posso oferecer atendimento melhor na cena do acidente, com recursos limitados, do que minhas crianças e eu recebemos em um hospital básico, alguma coisa esta errada com o sistema e o sistema tem que ser mudado. Contudo, colocando de maneira simples, nós temos primeiro que ajudar a treiná-los antes de acusá-los". E este passou a ser o objetivo principal do ATLS ${ }^{1,2}$.

Desde esta época, e após a adoção do ATLS pelo American College of Surgeons (ACS), já ocorre- 
ram mais de 25.000 cursos no mundo, com cerca de 500.000 participantes. Todo ano, cerca de 24.000 médicos recebem o treinamento em cerca de 25 diferentes países. Embora existam ainda alguns críticos, a aceitação maciça, rápida e disseminada da proposta tornou difícil uma contra-argumentação contundente e incontestável. $O$ fato é que esta proposta de atendimento organizou um grande volume de conhecimento prévio, criando uma linguagem nova e específica, atualmente adotada pela maioria dos emergencialistas e cirurgiões de trauma ao redor do globo ${ }^{3}$.

No ensino do atendimento ao traumatizado, assim como em outras áreas da medicina, a relação entre oportunidade de aprendizado prático e a assistência adequada é complicada e muito prejudicada pela situação de emergência, bem como pelo grande potencial de morte de cada caso. Cada procedimento deve ser feito uma única vez e pelo membro mais experiente da equipe. Para suprir as necessidades de treinamento de alunos e jovens médicos, sem colocar em risco a população, cada vez mais se tem lançado mão de instrumentos de simulação, sejam eles modelos animais, artificiais, virtuais ou atores treinados. Este é um dos princípios do ATLS e um dos motivos do seu reconhecimento mundial.

Neste artigo descreveremos os principais pontos que tornaram o ATLS uma proposta didática tão bem aceita, apresentando, também, parte da experiência desenvolvida no núcleo da Faculdade de Medicina de Ribeirão Preto.

\section{1- OBJETIVOS DO ATLS}

O principal objetivo do curso é treinar médicos que não participam regularmente de atendimento a traumatizados, e que trabalham em pequenos hospitais, unidades de saúde e na zona rural, para o atendimento inicial ao traumatizado, diagnóstico e tratamento de lesões que levam a morte rapidamente, e para obter a melhor estabilização possível, permitindo a transferência adequada da vítima para um local de referência para tratamento definitivo. Após os primeiros anos de sua aplicação, alguns trabalhos provaram a eficiência desta abordagem ${ }^{4,5,6}$, embora esta opinião não tenha sido unânime ${ }^{7}$.

Ao longo dos anos, o curso e toda a metodologia tornaram-se a linguagem comum entre as equipes de atendimento a emergências. Provou ser adequado também para os grandes centros de trauma, facilitando não somente o cuidado das vítimas, como também comunicação entre os diversos níveis de atendimen- to ${ }^{8}$. Nos Estados Unidos, Canadá e em alguns países da Europa, a aprovação em um curso ATLS, e a manutenção periódica deste título, é requisito básico para exercer a medicina, não somente em hospitais de emergência, como também em hospitais comunitários e para médicos de família.

\section{2- METODOLOGIA DE ENSINO DO ATLS}

Para atingir seu objetivo, o ACS reuniu um grande número de especialistas e pesquisadores para estudar e desenvolver um método, o mais seguro e padronizado possível, para o atendimento inicial a traumatizados graves. Partiu-se do princípio da necessidade de um curso eminentemente prático, com exposição a técnicas manuais, à semelhança do curso "Advanced Cardiac Life Support". Para manter o conteúdo do curso atualizado, o ACS faz revisões sistemáticas a cada 4 anos, sempre à procura das mais novas evidências científicas para cada procedimento e conduta propostos, recebendo sugestões da comunidade mundial de instrutores. As novidades são sistematizadas em uma nova edição do manual de alunos e difundidas para todos os núcleos ATLS no mundo9 .

O curso é realizado de maneira intensiva e concentrada, descrito como método de "imersão" no assunto, restrito a 16 alunos graduados em medicina ou em odontologia buco-maxilo-facial. A programação geralmente é realizada em dois dias (cerca de 20 horas de atividade no total), sendo ministrada através de aulas teóricas tradicionais, demonstrações, discussões em grupo, práticas em modelos animais e em bonecos, e simulação de atendimento em traumatizados (atores treinados e maquiados para simulação de cenários). Todo material didático é padronizado e distribuído pelo ACS, de maneira a garantir que a reprodução do curso seja rigorosamente semelhante em qualquer país.

O manual com todo o conteúdo teórico, programação e orientação para aulas práticas é distribuído aos alunos com pelo menos 30 dias de antecedência, com a recomendação explícita de que deve ser estudado profundamente, antes do início do curso ${ }^{9}$. Adicionalmente é distribuída uma prova com 40 questões com respostas de múltipla escolha, que deve ser realizada como pré-avaliação do conhecimento.

\section{1- Aulas teóricas}

As aulas teóricas são desenvolvidas nas primeiras horas de curso, com o objetivo de proporcionar uma rápida revisão do assunto previamente estudado no manual, tentando realizar um nivelamento de co- 
nhecimento entre os alunos. A orientação do ACS para os instrutores é de apresentarem o assunto de maneira interativa, procurando conseguir o maior envolvimento possível da platéia, proporcionando um bom momento para o esclarecimento de dúvidas. $\mathrm{O}$ entusiasmo e conhecimento profundo do assunto são atributos fundamentais dos instrutores do ATLS. No entanto, frequientemente, estas aulas são objeto de queixas por parte dos alunos que, ao final do curso, têm a sensação de um maior aproveitamento durante as aulas práticas e simulações.

\section{2- Aulas práticas e discussões}

O ATLS é especialmente focado neste tipo de oportunidade didática. Em primeiro lugar o grupo de alunos é subdivido, respeitando-se a proporção 4:1 (alunos/instrutor). São desenvolvidas práticas baseadas na avaliação de radiografias reais (tórax, coluna cervical, pélvis, tomografia computadorizada de crânio), manequins (bonecos simulando trauma de crânio, acesso venoso, vias aéreas), animais anestesiados (drenagem de tórax, cricotireoidostomia, lavado peritoneal diagnóstico, punção pericárdica), material de resgate e imobilização (pranchas, talas).

As discussões também são realizadas em grupos de 4 ou 8 alunos e são baseados em material teórico previamente distribuído, como cenários de catástrofes para o treinamento das decisões em situações que necessitam de triagem. Também o pré-teste é discutido em grupo, ainda no primeiro dia, procurando esclarecer as questões com maior índice de erros.

\section{3- Demonstrações}

Pelo menos em dois momentos do curso o recurso da demonstração de um atendimento simulado é realizado. No primeiro dia, uma simulação de um atendimento inicial ao traumatizado é encenada ou reproduzida por vídeo para os alunos, abordando principalmente as lesões com risco de morte (instrutor, paciente/ator, enfermeiro). Procura-se proporcionar uma visão, o mais real possível, da proposta do ATLS colocada em prática. No segundo dia outra demonstração é proporcionada, procurando apresentar um atendimento completo ao traumatizado, e mais uma vez gerando um momento de discussão e esclarecimento. Tais demonstrações cumprem papel fundamental no curso, pois agregam todo o conhecimento teórico transmitido em uma situação "real" de aplicação, muitas vezes esclarecendo conceitos até então muito abstratos.

\section{4- Cenários simulados - Atendimento Inicial ao Traumatizado}

Do ponto de vista do treinamento prático, o curso culmina com a participação dos alunos em cenários completos e simulados de atendimento a vítimas de trauma ${ }^{10}$. Cada aluno é exposto a 3 casos distintos, escolhidos dentre 20 scripts pré-definidos, com vítimas de trauma preparadas em todos os detalhes. $\mathrm{O}$ conjunto de situações procura abranger inúmeras possibilidades de traumas graves, e são previamente escolhidas pela direção do curso, seguindo-se a maquiagem e encenação dos atores e enfermeiros. Cada equipe (instrutor, ator e enfermeiro) conhece profundamente seu "caso", toda história do acidente, as lesões existentes, a condição clínica da vítima e as necessidades terapêuticas. A evolução do caso dependerá da conduta do aluno/médico, podendo agravar-se diante de uma opção equivocada. Uma sequiência de erros ou uma falha grave pode levar o paciente/ator ao óbito. De maneira geral não há improvisações e todas as possibilidades são previstas no script do caso.

Em uma escala de rodízio uma dupla de alunos é exposta ao primeiro cenário. Um dos alunos será o médico atendente e o outro um observador, que ao final de 20 minutos terá a oportunidade de avaliar e comentar o atendimento do colega. No segundo cenário, invertem-se os papéis. No terceiro cenário, cada aluno fará o atendimento sozinho durante 15 minutos, e será avaliado sobre a aplicação dos conceitos desenvolvidos durante todo o curso.

\section{5- Avaliação e aprovação}

A avaliação do curso ATLS se dá durante todo o decorrer do curso, em particular nas estações práticas. O instrutor emite um conceito ao final de cada atividade, que comporá a decisão final de aprovação. Contudo, no final do segundo dia uma segunda prova teórica de múltipla escolha é ministrada, cujo resultado mínimo a ser atingido é de $80 \%$. Por fim, e com maior peso, avalia-se o resultado do último atendimento simulado. Para esta avaliação, cada cenário possui um conjunto mínimo de condutas e procedimentos a serem "realizados". Obviamente, a sobrevivência do paciente é um critério comum a todos os casos, mas a demonstração da compreensão e aplicação dos conceitos desenvolvidos ao longo do curso são fundamentais. Em particular a aplicação do conceito de prioridades na seqüência do diagnóstico e tratamento das lesões com risco de morte iminente é um critério maior de aprovação. 


\section{3- ATLS NO BRASIL E EM RIBEIRÃO PRETO}

O primeiro curso ATLS foi ministrado no Brasil em 1989, na Faculdade de Medicina de São Paulo, da Universidade de São Paulo. A partir deste momento, com incentivo e administração central deste núcleo, o curso se difundiu rapidamente. Até 2004, mais de 20.000 médicos haviam sido treinados no país.

A criação do núcleo de Ribeirão Preto ocorreu durante o ano de 1994, com a realização do primeiro curso quase que exclusivamente reservado aos docentes do Departamento de Cirurgia, Ortopedia e Traumatologia. Durante os 10 anos que se seguiram, a Disciplina de Cirurgia de Emergência e Trauma realizou 102 cursos ATLS, formando cerca de 1.650 médicos.

Desde o início do núcleo, houve a decisão de adotar o curso como parte do treinamento dos médicos residentes do Departamento de Cirurgia e Anatomia (DCA). Desta maneira do total de alunos no período, $11 \%$ foram médicos residentes da cirurgia geral, ainda no seu primeiro ano de treinamento. Com esta proposta, pelo menos as últimas 13 gerações de cirurgiões formados no DCA, foram certificados pelo ACS para o atendimento inicial ao traumatizado. Do total de alunos $37,7 \%$ eram médicos residentes de todas as especialidades.

Ainda neste período, a grande procura pelo curso se deu por cirurgiões $(57,6 \%)$ procedentes de Ribeirão Preto $(46,1 \%)$. Mas a abrangência do núcleo de Ribeirão Preto atingiu não apenas as cidades da região como outras cidades de estados vizinhos (Londrina, Uberaba, Uberlândia, Juiz de Fora, Brasília). O grupo participou também na formação e supervisão de novos núcleos ATLS em Londrina, Uberaba, Brasília e São José do Rio Preto, dando continuidade à proposta do ACS de difusão e reprodução do curso.

Também no nosso meio, foi possível a realização de trabalhos científicos que demonstraram avanços no atendimento ao traumatizado após a adoção da metodologia do ATLS ${ }^{11}$. Estes bons resultados e a aceitação do curso por parte do corpo docente colaboraram com a elaboração de cursos para graduação da Faculdade de Medicina de Ribeirão Preto, altamen- te influenciados pela filosofia do ACS. Atualmente, conceitos do ATLS são ministrados desde o curso de Primeiros Socorros, no segundo ano da graduação e são complementados por um curso completo no primeiro ano do internato, imediatamente antes do estágio nas enfermarias da Disciplina de Cirurgia de Urgência e Trauma.

\section{4- CONCLUSÃO}

O curso ATLS se tornou um método de abrangência e reconhecimento mundiais, para o ensino do atendimento inicial e a ressuscitacão do paciente traumatizado. $\mathrm{O}$ curso prima pelo uso de simulações de situações de atendimento e pelo uso de manequins e modelos práticos de ensino. Sua abordagem é tida como regra no meio médico-cirúrgico e, embora ainda alvo de algumas críticas, apresenta-se como o melhor método de ensino e de atendimento às vítimas de trauma grave ${ }^{3}$.

\section{AGRADECIMENTOS}

À toda equipe de enfermagem da Unidade de Emergência do Hospital das Clínicas da FMRPUSP pela colaboração na execução do curso ATLS, como também na sua aplicação no dia-a-dia da sala de trauma.

À Beatriz Solange Bordon e Maria Alice França secretárias do curso ATLS no núcleo de Ribeirão Preto, pela dedicação e zelo na organização deste curso durante todos estes anos.

Aos Instrutores do curso ATLS do núcleo de Ribeirão Preto: Ajith Kumar Sankarankutty, Carlos Augusto F. Molina, Edwaldo Edner Joviliano, Flávio Marson, Gerson Alves Pereira Junior, Gustavo Ribeiro de Oliveira, João Batista Cardoso, José Ivan de Andrade, José Luiz Romeo Boulosa, Koji Tanaka, Leonardo Kruschewsky, Luis Donizeti da Silva Stracieri, Luiz Renato Ricci Camperoni, Maurício Kfuri, Ney Rodrigues Alves Dezotti, Omar Féres, Orcina Fernandes Duarte Joviliano, Paulo César Espada, Ricardo Luiz Santos Garcia, Sandro Scarpelini, Sérgio Luiz Brasileiro Lopes, Sidney José Mori Fernandes, Walther de Oliveira Campos Filho. 
Scarpelini S, Andrade JI. Simulation of patients - Life Support Courses - Advanced Trauma Life Support. Medicina (Ribeirão Preto) 2007; 40 (2): 199-203.

Abstract: During the last 30 years, the Advanced Trauma Life Support course from the American College of Surgeons became the worldwide reference in the trauma initial care education. Since the first course in Nebraska, 1978, it has been reproduced in more than 25 countries, due to its objective approach, dynamic and skilled based teaching of the life threatening situations of the trauma patient and the systematic training of the main surgical skills, fundamental to the success of the care. This course, based in artificial simulators, animal models, and actors seems to be a very efficient tool to the emergency medical education.

Keywords: Education, Medical. Simulation. Trauma. ATLS. Advanced Trauma Life Support.

\section{REFERÊNCIAS}

1 - Styner JK. The birth of Advanced Trauma Life Support (ATLS). Surgeon 2006;4:163-5.

2 - Collicott PE. Advanced Trauma Life Support (ATLS): past, present, future. 16th Stone Lecture, American Trauma Society. J Trauma 1992;33:749-53.

3 - Carley S, Driscoll P. Trauma education. Resuscitation 2001;48:47-56.

4 - Collicott PE, Hughes I. Training in advanced trauma life support. JAMA 1980;243:1156-9.

5 - Ali J, Howard M. The Advanced Trauma Life Support Program in Manitoba: a 5-year review. Can J Surg 1993;36:181-3.

6 - Ali J, Adam R, Butler AK, Chang H, Howard M, Gonsalves D et al. Trauma outcome improves following the advanced trauma life support program in a developing country. J Trauma. 1993;34:890-8.
7 - Vestrup JA, Stormorken A, Wood V. Impact of advanced trauma life support training on early trauma management. Am J Surg 1988;155:704-7.

8 - Gwinnutt CL, Driscoll PA. Advanced trauma life support. Eur J Anaesthesiol 1996;13:95-101.

9 - Committee on Trauma. Advanced Trauma Life Support. Student Course Manual. Chicago: American College of Surgeons; 2004.

10 - Ali J, Cohen RJ, Gana TJ, Al-Bedah KF. Effect of the Advanced Trauma Life Support program on medical students' performance in simulated trauma patient management. $\mathrm{J}$ Trauma 1998;44:588-91.

11 - Campos Filho WO. Impacto da implementação de um programa de treinamento de recursos humanos (Advanced Trauma Life Support) sobre a qualidade da atenção prestada às vítimas de traumatismo, no âmbito hospitalar. [Dissertação de Mestrado], Ribeirão Preto: Faculdade de Medicina de Ribeirão Preto - USP; 1999. 